\title{
Comparison of Downstream Transmitters for High Loss Budget of Long-Reach 10G-PON
}

\author{
Zhengxuan Li, Lilin Yi*, Weisheng Hu \\ State Key Lab of Advanced Optical Communication Systems and Networks, Shanghai Jiao Tong University, Shanghai 200240, China, \\ *Email: lilinyi@sjtu.edu.cn
}

\begin{abstract}
A comparison among different transmitters was made by evaluating the sensitivities under various launch powers and reaches. Experimental results indicate that directly-modulated laser based transmitters provide higher loss budget for long reach 10G-PON.

OCIS codes: (060.2330) Fiber optics communications; (060.2360) Fiber optics links and subsystems
\end{abstract}

\section{Introduction}

For the long-term goal of passive optical network (PON) systems, integration of metro and access networks is becoming a new trend because the CAPEX and OPEX of service providers will be reduced if large number of central offices (COs) is reduced or consolidated [1]. However, the consolidation of COs requires a growth in the reach and split ratio of PONs, which calls for a high loss budget. For a truly passive network, no repeater is allowed in the fiber plant, so the launch power of the transmitter and sensitivity of the receiver determine the link loss budget. The upstream receiver sensitivity can be significantly improved by coherent detection or hybrid Raman/Erbium-doped fiber amplifier, and in most of cases the link loss budget is limited by the downstream direction. Symmetric 10G-PON with loss budget up to $53 \mathrm{~dB}$ (considering the FEC limit BER of $3.8 \times 10^{-3}$ ) has been achieved by using coherent digital receiver in optical network unit (ONU) [2]. However, for practical application, direct detection is preferred considering the ONU cost. Loss budget of $51 \mathrm{~dB}$ was achieved by using semiconductor optical amplifier (SOA) as preamplifier in ONU [3], which will still increase the ONU cost. Increasing the downstream launching power is another solution to improve the downstream loss budget, however high power induced signal distortion due to nonlinear effects in fiber such as stimulated Brillouin scattering (SBS) and self-phase modulation (SPM) limits the maximal launching power. In most of previous long reach 10G-PON demonstrations, external modulation using Mach-Zehnder modulator (MZM) or electro-absorption-modulated laser (EML) were adopted, which have been considered with superior performance compared with direct modulation laser (DML).

In this paper, we evaluated the link loss budget in long reach 10G-PON of several commonly used transmitters including MZM, EML and DML. By measuring their sensitivities under different launch powers and reaches, we show that DML based transmitters have higher tolerance to fiber dispersion and nonlinearity, which is particularly suitable for long reach PONs. 50-dB loss budget can be achieved for 165-km reach 10G-PON by using DML and APD as transmitter and receiver. Direct modulation and direct detection simplify the network structure and cut down the cost, providing a cost-effective candidate for practical applications.

\section{Experimental setup and results}

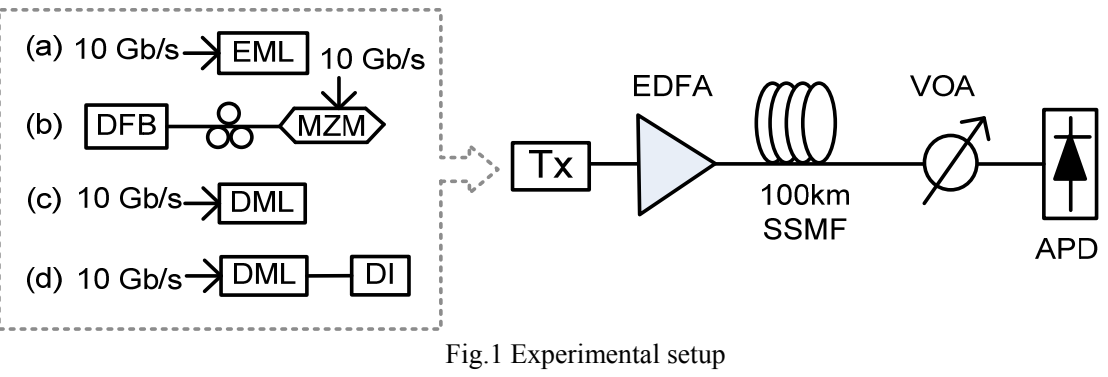

The comparison of different downstream transmitters was carried out under 100-km standard single mode fiber (SSMF) transmission case. Fig.1 depicts the experimental setup. All the transmitters operating at $1542 \mathrm{~nm}$ are driven by $10-\mathrm{Gb} / \mathrm{s}$ PRBS data with a word length of $2^{31}-1$. An erbium-doped fiber amplifier (EDFA) with an output power up to $23 \mathrm{dBm}$ was used to boost the signal power before being launched into the $100-\mathrm{km}$ fiber. A variable optical attenuator (VOA) was used to imitate the optical splitter and for sensitivity measurement. Four 10-Gb/s transmitters using EML, MZM, DML and DML followed by a delay interferometer (DI) to imitate chirp-managed laser (CML) were used for comparison. For high launch power scenario, an important nonlinear impairment is SBS. From the optical spectral point of view, both EML and MZM based NRZ-OOK format has a strong carrier component that can easily reach the SBS threshold and cause signal distortion. Inversely, the optical spectra of both DML and CML are carrier-less, which make these transmitters especially suited for 
high-launch power applications. DPSK and duobinary formats generated by MZM are also carrier-less, but they are not in the scope of comparison due to higher cost. Besides, SPM is another significant factor that impairs signal when the pulse peak power is high. Signal with a low extinction ratio (ER) has superiority in this respect because the "1" level has a relatively lower power than in high ER case. Finally, fiber dispersion caused signal impairment is also inevitable for long reach transmission.

Fig. 2 gives the experimental results in terms of sensitivity as a function of launching power, which confirms the previous prediction. Note that sensitivity in this paper refers to the power received by the APD with bit error rate $(\mathrm{BER})$ of $3.8 \times 10^{-3}$. From the results we can see that for EML, the sensitivity falls down rapidly when the launch power exceeds $14 \mathrm{dBm}$, resulting in a highest loss budget of $45 \mathrm{~dB}$ as marked in Fig. 2(a). Fig. 2(b) show the results for MZM transmitter at two different ER cases. When the signal has an ER of $11 \mathrm{~dB}$, the results are similar with the EML case. As we adjusted the Vpp of the driven signal and decreased the ER to $4 \mathrm{~dB}$, the sensitivity degradation slope becomes much gentle due to the reduction of pulse peak power. As a result, the best loss budgets are $47 \mathrm{~dB}$ and $47.5 \mathrm{~dB}$ in the 11-dB and 4-dB ER cases respectively. However, for DML and CML, the situation is different as shown in Fig. 3(c) and (d). Generally speaking, DML is unsuitable for high data rate, long distance fiber transmission due to the strong frequency chirp. The chirp broadens the optical spectrum and distorts signal during fiber transmission due to the chromatic dispersion. However, when the fiber is long enough, the fiber dispersion will firstly distort the signal and then convert the frequency modulation into intensity modulation, which is known as dispersion supported transmission (DST) technique [4]. Also, the low ER $(2 \sim 3 \mathrm{~dB})$ of the signal makes it more robust to SPM effect, which is quite suitable for high launch power application. The measured results of DML under various launch powers are shown in Fig. 3(c). We can see that due to the interaction between SPM and dispersion, the sensitivity was improved with the increase of launch power until the launch power exceeds $21 \mathrm{dBm}$, providing a highest loss budget of $51 \mathrm{~dB}$. Except for DST, spectral reshaping filter is more widely used to improve the transmission performance of DML, which is also known as CML [5]. By narrowing down the optical spectrum as well as increasing the ER, higher dispersion tolerance is obtained, which allows for long distance transmission. We used a DI as a notch filter instead of a bandpass filter commonly used in CML to realize the spectral filtering. Similar with DML, the carrier-less spectra show higher tolerance to SBS. But as the ER is enhanced to $\sim 10 \mathrm{~dB}$, the SPM effect is stronger. The sensitivity decreases at a lower launching power of $20 \mathrm{dBm}$ as shown in Fig. 2(d). Note that for all the transmitters, when the launching power exceeds $22 \mathrm{dBm}$, the signals are so severely impaired that we cannot get a BER lower than $3.8 \times 10^{-3}$.

Table. 1 summarizes the results. It can be concluded that for large-split, long-reach PONs, DML and CML show better performances than other transmitters. In the following section, an investigation on DML and CML was performed to evaluate their performances under various transmission distances for further evaluating their application values in practical situations.
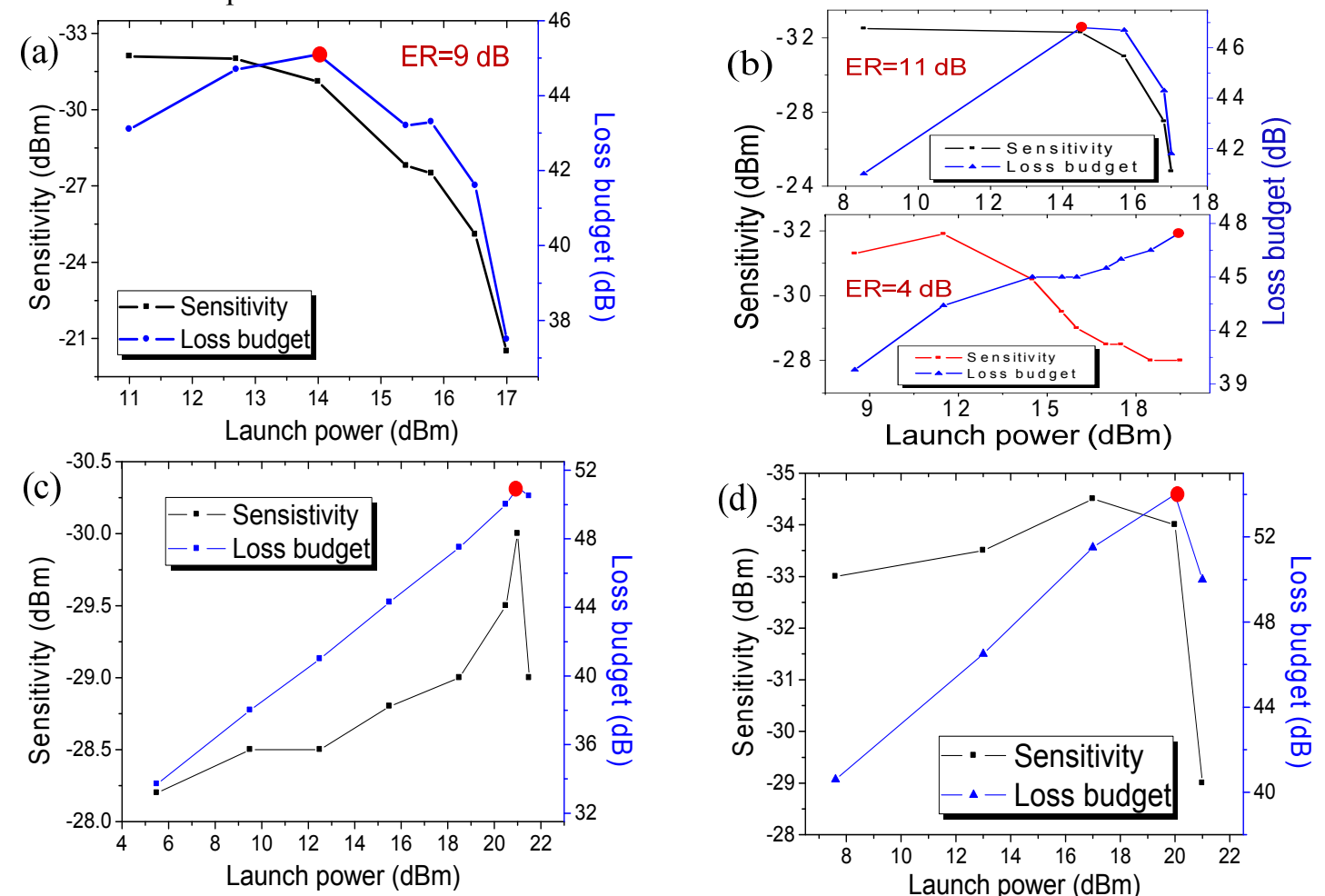

Fig.2 Sensitivity of downstream signal at BER of 1e-3 as a function of launch power using (a) EML (b) OOK modulation using MZM (c) DML and (d) CML as transmitters 
Table.1 Summary of transmitter performances

\begin{tabular}{|c|c|c|c|c|c|}
\hline Transmitter & $\begin{array}{c}\text { Maximal Launching } \\
\text { power }(\mathbf{d B m})\end{array}$ & $\begin{array}{c}\text { Sensitivity } \\
\mathbf{( d B m})\end{array}$ & $\begin{array}{c}\text { Loss budget } \\
(\mathbf{d B})\end{array}$ & $\begin{array}{c}\text { Split } \\
\text { ratio }\end{array}$ & $\begin{array}{c}\text { Margin } \\
(\mathbf{d B})\end{array}$ \\
\hline $\mathrm{EML}$ & 14 & -31 & 45 & 256 & 1 \\
\hline $\mathrm{MZM}(\mathrm{ER}=11 \mathrm{~dB})$ & 16 & -31 & 47 & 512 & 0 \\
\hline $\mathrm{MZM}(\mathrm{ER}=4 \mathrm{~dB})$ & 19.5 & -28 & 47.5 & 512 & 0.5 \\
\hline $\mathrm{DML}$ & 21 & -30 & 51 & 1024 & 1 \\
\hline $\mathrm{DML}+\mathrm{DI}$ & 20 & -34 & 54 & 2048 & 1 \\
\hline
\end{tabular}

\section{Transmission evaluation of DML and CML under different reaches}

The transmission performances of DML and CML were further investigated by measuring the receiver sensitivities after various fiber transmission distances as shown in Fig.3. Note that the sensitivities were measured at launching power of $21 \mathrm{dBm}$ and $20 \mathrm{dBm}$ for DML and CML respectively. The eye diagrams are exhibited in the insets of Fig. 3. For non-reshaping case, the eye diagram was firstly distorted during fiber transmission and then became clear again after propagating $75 \mathrm{~km}$. A clear eye opening was obtained even after 165-km SMF transmission, which was in coincidence with the DST theory. By using DI for spectral reshaping, the sensitivities can be further improved by $19 \mathrm{~dB}, 12 \mathrm{~dB}, 7$ $\mathrm{dB}, 4 \mathrm{~dB}$ and $2 \mathrm{~dB}$ in $25 \mathrm{~km}, 50 \mathrm{~km}, 75 \mathrm{~km}, 100 \mathrm{~km}$ and $165 \mathrm{~km}$ cases respectively. The improvement caused by spectral filtering is obvious when the fiber length is less than $100 \mathrm{~km}$. But the sensitivity differences decrease as the transmission distance exceeds $100 \mathrm{~km}$, meaning that DML can well support long distance transmission even without spectral filtering. At $165-\mathrm{km}$ reach, the loss budget of $50 \mathrm{~dB}$ is only $1 \mathrm{~dB}$ less than that of the CML based link. And for short distance cases, although the sensitivity is low, the split ratio can also be as high as 1:1024 because of the lower transmission loss of the shorter fiber link. Tab. 2 provides the loss budget evaluation in different transmission distance cases using DML as the downstream transmitter. Taking the transmission performance, the device stability and cost factor into account, DML can be a good option for 10G-PON application, especially in long-reach scenario.

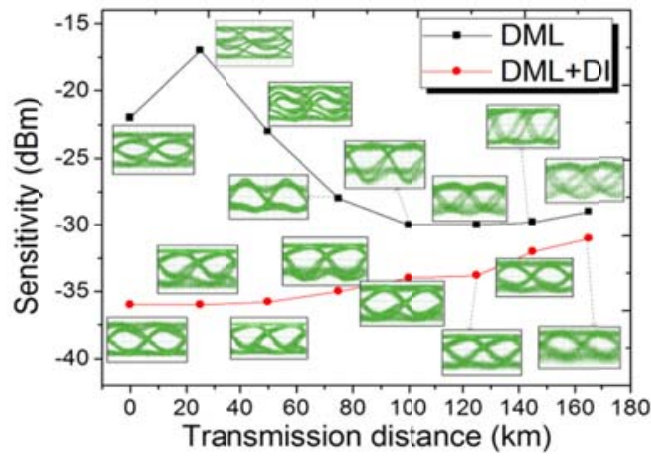

Tab.2 DML loss budget evaluation for different reaches and split ratio

\begin{tabular}{|c|c|c|c|c|}
\hline $\begin{array}{c}\text { Reach } \\
(\mathbf{k m})\end{array}$ & $\begin{array}{c}\text { Loss } \\
\text { budget } \\
\text { (dB) }\end{array}$ & $\begin{array}{c}\text { Fiber } \\
\text { loss } \\
\text { (dB) }\end{array}$ & $\begin{array}{c}\text { Split } \\
\text { ratio }\end{array}$ & $\begin{array}{c}\text { Margin } \\
\text { (dB) }\end{array}$ \\
\hline 25 & 38 & 5 & 1024 & 3 \\
\hline 50 & 44 & 10 & 1024 & 4 \\
\hline 75 & 49 & 15 & 1024 & 4 \\
\hline 100 & 51 & 20 & 1024 & 1 \\
\hline 125 & 51 & 25 & 256 & 2 \\
\hline 145 & 50.8 & 29 & 128 & 0.8 \\
\hline 165 & 50 & 33 & 32 & 2 \\
\hline
\end{tabular}

Fig.3 Sensitivities and eye diagrams of DML and CML under various transmission distances

\section{Conclusion}

The receiver sensitivities of different transmitters were measured under high launching power and long distance transmission conditions. Experimental results demonstrate that DML features a high tolerance to fiber nonlinearities such as SBS and SPM. Moreover, the DST technique makes the long distance transmission possible without any dispersion compensation. 50-dB loss budget can be achieved for reach between $100 \mathrm{~km}$ and $165 \mathrm{~km}$ which proves DML to be a cost-effective option as downstream transmitter for long-reach, large-split 10G-PON applications.

\section{References}

[1] D. P. Shea and J. E. Mitchell, “Long-reach optical access technologies," IEEE Netw., vol. 21, no. 5, pp. 5-11, Sep./Oct. 2007. [2] D. Lavery, E. Torrengo, and S. Savory, "Bidirectional $10 \mathrm{Gbit} / \mathrm{s}$ long reach WDM-PON using digital coherent receivers," in Proc. OFC/NFOEC,Mar.2011, Paper OTuB4.

[3] D. Qian, E. Mateo, and M-F. Huang, "A $105 \mathrm{~km}$ reach fully passive 10G-PON using a novel digital OLT," in Proc. ECOC., Sep. 2012, Paper Tu.1.B.2.

[4] B. Wedding, B. Franz, and B. Junginger, "10-Gb/s optical transmission up to $253 \mathrm{~km}$ via standard single-mode fiber using the method of dispersion-supported transmission”, J. Lightw. Technol., vol. 12, no. 10, pp. 1720-1727, Oct. 1994.

[5] S. Chandrasekhar, C. R. Doerr, L. L. Buhl, Y. Matsui, D. Mahgerefteh, X. Zheng, K. McCallion, Z. Fan, and P. Tayebati, "Repeaterless transmission with negative penalty over $285 \mathrm{~km}$ at $10 \mathrm{~Gb} / \mathrm{s}$ using a chirp managed laser," IEEE Photon. Technol. Lett. 17, 2454-2456 (2005). 